\title{
Effects of spatial order and inter-item interval on recall of temporal order'
}

Questions left over from an earlier investigation of recall of temporal order were taken up in three experiments. Recall was facilitated when the stimulus objects had a unidimensional spatial ordering that was perfectly correlated with the ir temporal ordering. Recall after a sequence with a 3-sec. inter-item interval did not differ significantly from recall after a sequence with a 6-sec. interval. The results of these and the previous experiments are taken to favor the hypothesis of an "internal counter."

In three experiments performed some years ago (Berlyne, 1960), Ss either heard a sequence of 16 words or saw a sequence of 16 objects. Pairs of items from the sequence were then presented, and Ss had to indicate which item had occurred later than the other.

The main findings were as follows: (1) Whether the original sequence was presented once, three times, or six times, recall of temporal order was significantly above chance. (2) Scores were significantly higher after three or sixpresentations of the sequence than after one. (3) There was a significant serial-position effect. (4) Recall of temporal position was significantly better when two items had been separated by seven items in the original sequence than when they had been adjacent. (5) Whether items were taken from the first or second half of the sequence made no difference. (6) Whether the items in the sequence were presented at the same spatial location or at differing locations, uncorrelated with temporal positions, made no difference.

Among various hypotheses that were considered, the findings were deemed to favor the view that learning of temporal order depends on either an "internal clock" or an "internal counter." It is assumed that, during exposure to a sequence of stimulus items, some internal variable changes linearly with either the time that has elapsed since the beginning of the sequence ( the "clock" hypothesis) or the number of items that have already been presented (the "counter" hypothesis). The value reached by the internal variable when a particular stimulus item is presented becomes associated with that item as an internal mediating response and produces a corresponding form of internal stimulation. Given the mediators associated with two stimulus items, $\mathrm{S}$ can distinguish with above-chance reliability which mediator represents a later temporal position. In other words, there exists a natural relation between the mediators, generating a mediated relation between the associated stimulus items (Berlyne, 1965, pp. 65-67).

The following three experiments were designed to clear up two outstanding questions.

\section{Experiment I}

In Experiment III in the previous series (Berlyne, 1960), recall of temporal order was not impeded by a spatial ordering that was uncorrelated with the temporal ordering. The present experiment was designed to ascertain whether recall is improved when the spatial ordering and the temporal ordering correspond.

Two grade-9 classes, of 28 anu 27 members respectively, were tested as groups in their classrooms. Two sequences of 16 objects were prepared, being as far as possible replicas of those used in the earlier experiment.

Ss were first told that they were to see a sequence of objects and should pay close attention to them, because they would later be asked questions about them. Sequence 1 was then exposed twice in succession, with intervals of 3 sec. separating consecutive objects. Immediately after this phase (Phase I), pairs of objects taken from Sequence 1 were presented in turn above the mid-point of the front edge of the teacher's desk (Phase II). Half of the pairs had been separated by seven items and half had been adjacent in Phase I. Earlier and later items appeared equally often on the right and on the left. Ss were instructed that, on seeing each pair, they must decide which of the two objects had appeared later than the other in Phase I and then to write the letter $L$ (denoting the left-hand object) or the letter $\mathrm{R}$ (denoting the right-hand object) on the corresponding line of the answer sheet. A similar procedure was then carried out with Sequence 2.

Two experimental treatments were used in Phase I. The Dispersed treatment consisted of presenting the first object at the extreme left (from S's point of view) of the front edge of the desk, the second object just to the right of it, etc. In the Central treatment, all objects were presented just above the mid-point of the front edge of the desk. One class had the Dispersed treatment for Sequence 1 and the Central treatment for Sequence 2, while the other class had the treatments in the opposite order.

The mean number of correct responses in Phase II (out of 16) was 11.2 for the Dispersed treatment and 10.3 for the Central treatment $(F=4.09, \mathrm{df}=\mathbf{1} / \mathbf{5 3}$, $\mathrm{p}<.025,1$ tail). It seems therefore that a correlation between spatial ordering and temporal ordering can facilitate recall of the latter. Feedback from overt or covert spatially distributed responses (e.g., head movements, eye movements, finger pointing) could presumably add distinctiveness to the cues provided by the internal clock or counter. 


\section{Experiment II}

If the clock hypothesis is corrent, the mediators associated with two temporal positions will be less distinct, and recall of temporal order will therefore be less accurate, when items succeed one another at a faster rate. If the counter hypothesis is correct, interitem interval should make no difference within broad limits. The procedure for Experiment II was exactly the same as that of Experiment $I$, except that the two treatments used in Phase I consisted, respectively, of leaving an interval of $3 \mathrm{sec}$. and an interval of $6 \mathrm{sec}$. between appearances of consecutive objects. All objects were presented above the midpoint of the table. One grade- 9 class of 22 members had the 3-sec. treatment with Sequence 1 and the 6-sec. treatment with Sequence 2. The opposite held for another grade- 9 class of 18 members.

The mean scores in Phase II were 11.0 with the 3-sec. treatment and 10.7 with the 6-sec. treatment. This difference did not approach significance and is, in any case, in the opposite direction to what the "clock" hypothesis would imply.

\section{Experiment III}

One possible objection to Experiment II is that the mean time elapsing between the appearance of an object in Phase I and its appearance as a member of a pair in Phase II was shorter with the 3-sec. treatment. It is therefore conceivable that the 6-sec. treatment made for better learning but that this advantage was masked by a longer retention interval and thus more forgetting. Experiment III was designed to overcome this difficulty.

The procedure was the same as in Experiment II except for the following differences. When items were presented at $3-3 e c$. intervals in Phase I there was a delay of $2 \mathrm{~min}$. between the end of Phase I and the beginning of Phase II, and the pairs presented in Phase II appeared for 2 sec. each at intervals of 6 sec. When the interitem interval was 6 sec. in Phase I, there was an interval of $2 \mathrm{~min}$. before Phase II, and the pairs in Phase II were presented for 2 sec. at intervals of $3 \mathrm{sec}$. The mean retention interval, i.e., the mean time elapsing between appearances of a particular object in Phase I and in Phase II, was thus equal for the two treatments. One class of 29 summersession undergraduates received the 3-sec. treatment for Sequence 1 and the 6-sec. treatment for Sequence 2, with the reverse arrangement for a second class of 12 undergraduates.

The mean scores were 11.3 for the 3-sec. treatment and $\mathbf{1 1 . 1}$ for the 6-sec. treatment. This difference is comparable with that obtained in Experiment II and does not approach significance.

The results of Experiments II and III thus favor the "counter" hypothesis over the "clock" hypothesis.

\section{References}

Berlyne, D. E. L'apprentissage sériel et les relations d'ordre. In D. E. Berlyne and J. Piaget (Eds.), Théorie du comportement et opérations (Etudes d'épistémologie génétique. XII). Paris: Presses Universitaires de France, 1960.

Berlyne, D. E. Structure and direction in thinking. New York: Wiley, 1965.

\section{Notes}

1. This investigation was supported by Research Grant MH-06324 from the National Institute of Mental Health, U. S. Public Health Service, and by a grant from the Ontario Institute for Studies in Education.

2. The author is indebted to Mrs. Judith Lewis and to Miss Sharon Lazare, who collected the data for Experiments I and II and for Experiment III respectively, as well as to Mr. L. A. Sivell, Principal of the W. A. Porter Collegiate Institute, Scarborough, Ontario, who kindly arranged for his students to serve as subjects. 